\title{
Determinación de factores de riesgo involucrados en diarrea neonatal bovina en fincas lecheras del trópico alto colombiano
}

\author{
Pardo, D. ${ }^{1}$; Oliver, O. ${ }^{2}$ \\ ${ }^{1}$ Universidad Antonio Nariño, Facultad de Medicina Veterinaria, Bogotá Colombia, Calle 58 A No 37, \\ Tel. $+57(1) 338$ 4960, Ext.133. ${ }^{2}$ Universidad Nacional de Colombia, Facultad de Medicina \\ Veterinaria y de Zootecnia. E-mail: dopardo@uan.edu.co
}

\begin{abstract}
Resumen
Pardo, D.; Oliver, O.: Determinación de factores de riesgo involucrados en diarrea neonatal bovina en fincas lecheras del trópico alto colombiano. Rev. vet. 26: 2, 124-130, 2015. Con el objetivo de determinar los factores predisponentes a la diarrea neonatal bovina (DNB) relacionados con el ternero, la madre y el manejo de la finca, en las primeras cinco semanas de vida, se realizó un estudio longitudinal en el que se seleccionaron por conveniencia 21 fincas lecheras y de producción mixta ubicadas en la Sabana de Bogotá. Se registraron las variables relacionadas con el manejo, alimentación, parto, la madre y el ternero recién nacido. Se utilizó como medida de asociación la prueba de Chi-cuadrado $(\mathrm{p}<0,05)$ y el modelo de regresión logística $(\mathrm{p}<0,10)$. Se efectuó el seguimiento de 620 animales nacidos en 21 fincas, de los cuales un $10,3 \%(n=64)$ presentó $\mathrm{DNB}$. Se encontró que los terneros procedentes de fincas medianas y pequeñas, el uso de fertilizantes diferentes a los químicos, prácticas de suplementación, vacunación contra complejo neonatal bovino, raza diferente a la Holstein en madres y terneros, ser machos, tener peso menor de $39 \mathrm{~kg}$ o mayor de $45 \mathrm{~kg}$, desinfección de ombligos con productos diferentes a los yodados, permanencia post-separación de la madre en sistema diferente a cuerda y estaca y método de suministro diferente al uso de balde, fueron los factores de riesgo asociados causalmente con la presentación de DNB. Los factores asociados con menor riesgo fueron: el suministro de concentrado a los terneros, aplicación de vacunas en la finca contra leptospirosis, complejo respiratorio bovino y vacuna triple bovina, prácticas de desparasitación con albendazoles a terneros y animales adultos, aplicación de terapia de secado y asistencia técnica de veterinarios y zootecnistas.
\end{abstract}

Palabras clave: ternero, diarrea neonatal, factores de riesgo, manejo.

\begin{abstract}
Pardo, D.; Oliver, O.: Determination of risk factors involved in bovine neonatal diarrhea in dairy farms from the Colombian high tropical zone. Rev. vet. 26: 2, 124-130, 2015. In order to determine the factors related to the calf, mother and farm management that predispose calves to present bovine neonatal diarrhea (DNB) in the first 5 weeks of life, a longitudinal study was conducted in which 21 dairy farms and mixed production located in the Sabana de Bogota were selected. The variables related to management, nutrition, calving, cow and newborn calf were recorded. Chi-square test $(\mathrm{p}<0.05)$ and the logistic regression models $(\mathrm{p}<0.10)$ were used as association measure. Calves $(\mathrm{n}=620)$ development was followed in the 21 farms, of which $10.3 \%(n=64)$ presented DNB. It was found that calves from medium and small size farms, using fertilizers different to chemicals, supplementation practices, immunization against neonatal bovine complex, breed of cows and calves different to Holstein, males, weight less than $39 \mathrm{~kg}$ or more than $45 \mathrm{~kg}$, use of disinfection products different to iodine, management system after mother separation different to stake system and the use of different feeding methods rather than the use of a bucket, were risk factors causally associated with the presentation of DNB. Factors associated with lower DNB risk were: providing concentrates to calves, vaccination against leptospirosis, bovine respiratory complex and bovine triple vaccine, deworming practices with benzimidazoles in calves and adult animals, cow dry therapy, and technical assistance by a veterinarian and animal husbandry technician.
\end{abstract}

Key words: calf, neonatal diarrhea, risk factors, management. 


\section{INTRODUCCIÓN}

La diarrea neonatal bovina (DNB) es una enfermedad compleja y multifactorial que ocurre como consecuencia de la interacción de factores relacionados con la vaca, el ternero, el estado inmune, las prácticas de manejo, los factores ambientales y los gérmenes enteropatógenos ${ }^{15,23}$.

Estudios realizados en Colombia indicaron variabilidad en los niveles de incidencia de DNB. En investigaciones previas realizadas por autores de la presente publicación (y colaboradores) se hallaron tasas del 2,56\% en terneros de 0 a 2 meses de vida del Departamento de Nariño (XIX Congress World Association for Buiatrics, 1995); se encontró un porcentaje del 6,8\% en terneros del Departamento del Meta (Tesis FMVZ$U N C, 1998)$; se registró una morbilidad del $37,5 \%$ en terneros de 0 a 3 meses de vida en el Departamento de Antioquía (Tesis FMVZ-UNC, 1998); se obtuvieron guarismos del $44,9 \%$ de incidencia en terneros de la Sabana de Bogotá (XXIII World Buiatrics Congress, Quebec, Canadá, 2005) y recientemente se identificaron agentes infecciosos asociados a DNB (Rev MVZ Córdoba 17: 3, 2012).

Se han considerado como factores de riesgo aquellos asociados al parto, la alimentación, la vacunación, el alojamiento y el manejo del calostro ${ }^{17}$, también otros como el estado inmunológico del ternero, el consumo de calostro por un solo día, ser hijos de vacas primerizas, sistemas de crianza a la intemperie o en grupos de diferentes edades, nacimiento en invierno o en verano donde las condiciones climáticas son adversas, mala higiene de los utensilios de alimentación del ternero como baldes, teteros (biberones) y de los sitios de alojamiento (terneriles), tamaño de las fincas ${ }^{4,8,24}$, la presentación de partos distócicos ${ }^{3,12}$, presencia de altos niveles de amonio, manejo profiláctico, tiempo de permanencia del ternero con la madre, la excreción de ooquistes de Cryptosporidium parvum ${ }^{24}$, la infección con rotavirus y la coinfección con más de un enteropatógeno ${ }^{1}$.

El objetivo del estudio fue determinar los factores que predisponen a la presentación de DNB relacionados con el ternero, la madre y el manejo de la finca, en el trópico alto colombiano.

\section{MATERIAL Y MÉTODOS}

Diseño experimental: Se realizó un estudio observacional combinado de cohortes y caso-control en 21 fincas de la Sabana de Bogotá, región situada a una altitud de $2.600 \mathrm{msnm}$, utilizando un muestreo por conveniencia. Fueron 18 lecherías especializadas, 2 sistemas doble propósito con predominio de raza Normando y una finca con actividades de atención de partos y cuidado de los terneros en sus primeras semanas de vida dentro de un programa de transferencia de embriones principalmente de raza Angus; las fincas fueron consideradas grandes cuando tenían una extensión mayor a 60 ha, medianas entre 30 y 60 ha y pequeñas con menos de 30 ha.
Recolección de la información: se realizaron encuestas a los propietarios y trabajadores, se revisaron registros existentes y se obtuvieron datos relacionados con el estado de salud, estado productivo, reproductivo y manejo general a nivel de finca y del ternero. Durante los 12 meses de estudio se registraron los partos en las 21 fincas y de cada uno se tomaron los datos relacionados con el manejo (sistema de alimentación, vacunación, vermifugación, alojamiento, terapia de secado) de la madre, la historia del parto y el estado del ternero recién nacido.

En los primeros 3 días de vida del ternero se realizó una visita para registrar los datos sobre su estado de salud, comportamiento, momento de incorporación, tiempo transcurrido entre el nacimiento y la primera toma de calostro y el manejo que cada ternero recibió desde ese momento en la finca. Cada ternero fue identificado de acuerdo al sistema utilizado en cada finca y su peso vivo fue obtenido con cinta bovinométrica Ovny ${ }^{\circledR}$.

Se realizaron visitas semanales de seguimiento de cada ternero hasta los 35 días de vida, registrando la presentación de DNB, definida como la evacuación de heces fluidas, frecuentes y abundantes, con anorexia y deshidratación. Inmediatamente se escogieron controles que fueron terneros de la misma finca sin signos clínicos de DNB u otra enfermedad, con una edad similar (diferencia menor a 7 días). Durante las primeras 72 horas de vida se tomó una muestra de sangre y en el suero se determinó el nivel de absorción de inmunoglobulinas calostrales de cada ternero por la técnica de precipitación con sulfito de sodio ${ }^{9}$.

Análisis estadístico: se utilizó como medida de asociación la prueba de chi-cuadrado (con un nivel de significancia de $\mathrm{p}<0,05)$. Posteriormente, para estimar el grado de asociación de los factores de riesgo con la presentación de la DNB, se utilizó el modelo de regresión logística $(p<0,10)^{7}$. En la interpretación de los odds ratio $(\mathrm{OR})$ se consideró que los valores inferiores a 1 son protectores frente a la presentación de enfermedad o muerte, mientras que los valores superiores a 1 indicaron el número de veces de riesgo para que se presenten estos eventos.

\section{RESULTADOS Y DISCUSIÓN}

Lapsos de aparición de la enfermedad. Se efectuó el seguimiento de 620 animales nacidos en las 21 fincas durante el año de estudio, de los cuales un 10,3\% $(n=64)$ presentaron signos clínicos de DNB, hallazgo similar al reportado recientemente en Nueva Zelanda con un 9,5\% en terneros hasta 21 días de edad ${ }^{1}$. La edad de presentación de diarrea fue la siguiente: en la primera semana de vida el $29,7 \%$ de los casos $(n=19)$, en la segunda el $40,6 \%(\mathrm{n}=26)$, en la tercera el $15,6 \%$ $(n=10)$, en la cuarta el $4,7 \%(n=3)$ y en la quinta el $9,47 \%$ $(n=6)$, como se detalla en la Figura 1.

La mayor cantidad de casos se registró en la segunda semana de vida, resultados que fueron similares a 


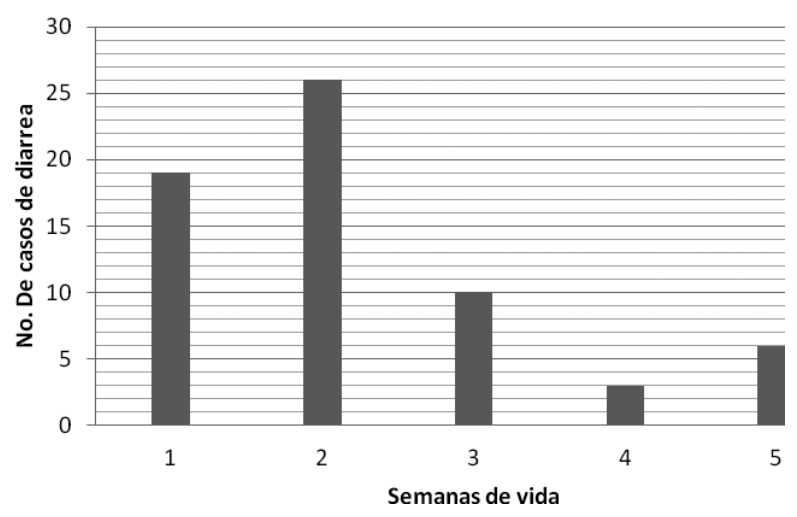

Figura 1. Edad de presentación de diarrea y tasa de terneros afectados. Los números de la escala indican cantidad de terneros afectados.

los observados en Suecia ${ }^{21}$, sin embargo en un estudio previo realizado en la Sabana de Bogotá se detectó una mayor presentación en la tercera semana de edad (datos propios). La aplicación de la prueba de $\mathrm{X}^{2}$ de manera univariada mostró 23 variables asociadas significativamente a la presencia de DNB $(\mathrm{p} \leq 0,1)$, las que fueron incluidas en el modelo de regresión logística ${ }^{16}$.

Variables asociadas a las características de la finca y al manejo. Como indica la Tabla 1, el riesgo de presentar la enfermedad aumentó en terneros de fincas medianas (entre 30 y 60 ha) y pequeñas (menores a 30 ha), frente a terneros pertenecientes a fincas grandes (mayores a 60 ha) (Tabla 2). En el caso de las fincas pequeñas el error estándar $(15,63)$ fue bastante alto, lo cual podría deberse a que el número de terneros procedentes de las cuatro fincas pequeñas fue tan solo de 32 animales, de los cuales enfermaron 3 animales (9,3\%), por tanto la representatividad del modelo no es concluyente en cuanto a la asociación con la enfermedad. En Colombia no se había reportado asociación entre el tamaño de las fincas y la presentación de diarrea.
Con respecto al tipo de alimentación, se presentó mayor probabilidad de enfermar en aquellas fincas donde habían praderas con avena, maíz y pasto azul orchoro (Dactylis glomerata); sin embargo estos forrajes se encuentran en las fincas en baja proporción. Este resultado ha sido reportado previamente y no hay una explicación clara sobre su implicación como factor de riesgo al igual que en el estudio realizado en el departamento del Meta donde se encontró asociación entre morbilidad y el pasto Braquiaria decumbens (datos propios).

Hubo un mayor riesgo de enfermar de DNB $(\mathrm{OR}=1,6)$ en las fincas que suplementaban los animales; los principales suplementos fueron: afrecho, semilla de algodón, melaza, harina de maíz, heno, henolaje, papa, silo, zanahoria, lípidos vegetales sobrepasantes ("rumipass"), carbonato de calcio, bicarbonato, complementos nutricios (mana) y soya. Anteriormente habíamos registrado algo similar para la suplementación con afrecho, melaza, torta de palmiste (palma africana) y forrajes ensilados ("silo"), sin embargo en el presente estudio hubo gran variedad en los productos utilizados y se deberían evaluar las características nutricionales, microbiológicas y el manejo de estos suplementos para poder explicar su papel como factor de riesgo.

En investigaciones anteriores se reportó que la alimentación con ensilaje de maíz se relacionaba con un mayor riesgo de enfermedad ${ }^{12}$, hallazgo que concuerda con los resultados aquí obtenidos, ya que este producto está dentro de los suplementos suministrados en las fincas y la explicación que sugieren de esta asociación es que este alimento incrementa transitoriamente el nivel de triglicéridos y de nitrógeno ureico sanguíneo, los cuales causarían el mayor riesgo. Adicionalmente, los hongos micotoxigénicos presentes en el ensilaje de maíz se han asociado con problemas de salud como toxicosis aguda, disminución en la productividad y mayor susceptibilidad a enfermedades en animales y seres humanos ${ }^{14}$.

Tabla 1. Resultados de la regresión logística para factores relacionados con la finca.

\begin{tabular}{llccc}
\hline variable & modalidad & odds ratio & intervalo confianza & error est. \\
\hline extensión finca & grande & - & - & - \\
& mediana & 5,1582 & $3,2524-8,1807$ & 1,2138 \\
& pequeña & 50,7836 & $27,7788-92,8396$ & 15,6318 \\
\hline presencia de pasto avena & no & - & - & - \\
& si & 15,37 & $10,3941-22,7300$ & 3,0681 \\
\hline otros pastos (azul orchoro, maíz) & no & - & - & - \\
& si & 14,2067 & $9,6503-20,9144$ & 2,8032 \\
\hline suplementación & no & - & - & - \\
& si & 1,6156 & $1,1765-2,2185$ & 0,2614 \\
\hline fertilización & química & - & - \\
& otra & 22,9808 & $15,1511-34,8568$ & 4,8845 \\
\hline frecuencia de fertilización & $0-2$ meses & - & - & - \\
& 2-6 meses & 1,892 & $1,2655-2,8287$ & 0,3882 \\
& mayor a 6 meses & 53,4546 & $29,8687-95,6653$ & 15,8537 \\
\hline asistencia técnica & veterinario & - & - & - \\
& vet.y zootecnista & 0,7052 & $0,5141-0,9673$ & 0,1137 \\
\hline
\end{tabular}


Tabla 2. Resultados de la regresión logística para factores relacionados con las madres.

\begin{tabular}{llccc}
\hline variable & modalidad & odds ratio & intervalo confianza & error est. \\
\hline otras vacunas & ninguna & - & - & - \\
& leptospirosis & 0,1802 & $0,1151-0,2820$ & 0,0412 \\
& complejo.resp. & 0,1277 & $0,0813-0,2006$ & 0,0294 \\
\hline albendazol madre & no & - & - & - \\
& si & 0,0835 & $0,0573-0,1218$ & 0,0161 \\
\hline albendazol ternero & no & - & - & - \\
& si & 0,069 & $0,0468-0,1017$ & 0,0137 \\
\hline vacuna triple bovina & no & - & - & - \\
& si & 0,2336 & $0,1670-0,3269$ & 0,04 \\
\hline vacuna complejo neonatal & no & - & - & - \\
& si & 29,7934 & $19,2807-46,0382$ & 6,6152 \\
\hline terapia de secado & no & - & - & - \\
& si & 0,0588 & $0,0395-0,0875$ & 0,0119 \\
\hline raza madre & Holstein & - & - & - \\
& Jersey & 4,8355 & $2,3356-10,0114$ & 1,7954 \\
& Pardo Suizo & 3,3473 & $0,6567-17,0619$ & 2,7815 \\
& Normando & 2,5105 & $0,5177-12,1739$ & 2,0223 \\
& Angus & 10,0392 & $0,6180-163,1$ & 14,2793 \\
\hline
\end{tabular}

Cuando se comparó la aplicación de fertilizantes químicos con una frecuencia inferior a los dos meses con prácticas diferentes o aumento del tiempo de aplicación, estas últimas representaron un mayor riesgo $(\mathrm{OR}=3,0)$. Un hallazgo similar fue reportado en el piedemonte llanero colombiano, en el que el uso del estiércol de gallina (gallinaza) como fertilizante fue un factor de riesgo de $\mathrm{DNB}^{19}$, en contraste con un estudio en la Sabana de Bogotá (datos propios) que halló que la fertilización química incrementó el riesgo de enfermar $(\mathrm{OR}=16, \mathrm{p}<0,05)$.

La asistencia técnica a cargo médicos veterinarios y zootecnistas $(\mathrm{OR}=0,7)$ fue un factor de protección y fue más favorable que la intervención únicamente del médico veterinario; este aspecto sugiere que contar con la asesoría de dos o más profesionales de áreas complementarias puede mejorar las características productivas y sanitarias de las fincas. En otras investigaciones se ha encontrado que el incremento en el número de visitas por parte del veterinario y la asistencia técnica permanente estuvieron asociados con una reducción en la incidencia de diarrea ${ }^{4}$.

Variables asociadas a las madres y su manejo. Como señala la Tabla 2, se encontró que cuando se aplicaron vacunas contra leptospirosis $(\mathrm{OR}=0,18)$, complejo respiratorio bovino $(\mathrm{OR}=0,13)$ y triple bovina (vacuna contra carbunclo sintomático, edema maligno y pasteurelosis) $(\mathrm{OR}=0,23)$ y también cuando se usó albendazol para desparasitar a las madres $(\mathrm{OR}=0,08)$ y a los terneros $(\mathrm{OR}=0,07)$, éstos tenían menor probabilidad de presentar DNB, mientras que hubo mayor probabilidad cuando se aplicaba vacuna para complejo neonatal bovino (vacunas que incluyen antígenos para Salmonella $s p$., Escherichia coli enterotoxigénica, rotavirus, coronavirus y Clostridium perfringens) $(\mathrm{OR}=29,8)$, en este caso es necesario evaluar si los genotipos de los agen- tes del producto biológico son los que están presentes en la zona y los protocolos de aplicación en las fincas de este tipo de vacuna.

Se ha reportado que las hembras vacunadas contra C. perfringens, diarrea viral bovina, rotavirus y coronavirus (pero no contra E. coli) presentan menor riesgo de DNB y se ha postulado que esto se debe a un buen manejo general de la finca y no a una relación específica con el tipo de vacuna ${ }^{12}$, también que la vacunación de vacas contra enteropatógenos de terneros, disminuye el riesgo de heces fluidas en los terneros ${ }^{1}$. En otro estudio (datos propios) se halló que el uso de albendazoles parece proteger contra la ocurrencia de DNB, pero no se encontró asociación con los programas de vacunación. En terneros se ha reportado una reducción de la presentación de DNB ocasionada por infecciones con rotavirus, al utilizar vacunas recombinantes en las vacas; estos estudios no han evaluado la aplicación combinada con otros agentes infecciosos ${ }^{5,20}$.

Los altos títulos de anticuerpos maternos modulan significativamente la respuesta de células secretoras de anticuerpos en el ternero y la administración de calostro con altos niveles de inmunoglobulinas reduce la presentación de signos pero no la infección por rotavirus ${ }^{18}$. Sin embargo, esto debe investigarse para otros agentes y aclarar el impacto de la vacunación de las madres para prevenir la enfermedad en los terneros.

La aplicación de terapia de secado en las vacas antes del parto hizo menos probable el riesgo de la presentación de $\mathrm{DNB}(\mathrm{OR}=0,06)$, lo cual puede asociarse a una reducción en la presentación de mastitis y por ende una mejor calidad microbiológica del calostro y de la leche administrada a los terneros. En Suecia se confirmó una asociación entre el conteo de células somáticas y la presentación de enfermedad respiratoria, pero no con DNB ${ }^{13}$. 
Tabla 3. Resultados de la regresión logística para factores asociados con el ternero.

\begin{tabular}{|c|c|c|c|c|}
\hline variable & modalidad & odds ratio & intervalo confianza & error est. \\
\hline \multirow[t]{5}{*}{ raza ternero } & Holstein & - & - & - \\
\hline & Jersey & 4,9907 & $2,3999-10,3784$ & 1,8643 \\
\hline & Pardo Suizo & 6,0705 & $1,4070-26,1915$ & 4,5282 \\
\hline & Normando & 2,9262 & $0,9208-9,2993$ & 1,7262 \\
\hline & Angus & 3,0627 & $1,3096-7,1627$ & 1,3276 \\
\hline \multirow[t]{2}{*}{ sexo ternero } & hembra & - & - & - \\
\hline & macho & 29,071 & $18,8477-44,8395$ & 6,4276 \\
\hline \multirow[t]{3}{*}{ peso } & entre $39-45 \mathrm{~kg}$ & - & - & - \\
\hline & menor a $39 \mathrm{~kg}$ & 2,1555 & $1,4719-3,1565$ & 0,4195 \\
\hline & mayor a $45 \mathrm{~kg}$ & 1,6927 & $1,1551-2,4805$ & 0,33 \\
\hline \multirow[t]{3}{*}{ fuente de calostro } & madre & - & - & - \\
\hline & otra vaca & 56,7777 & $7,3264-440,00$ & 59,318 \\
\hline & banco calostro & 12,0173 & $1,5915-90,7437$ & 12,3958 \\
\hline \multirow[t]{3}{*}{ desinfección ombligo } & yodado & - & - & - \\
\hline & ninguna & 5,9547 & $1,7908-14,2674$ & 2,6761 \\
\hline & eterol & 9,5927 & $3,9237-23,4526$ & 4,3754 \\
\hline \multirow[t]{4}{*}{ permanencia post-separación } & cuerda & - & - & - \\
\hline & terneril & 2,0884 & $1,1290-3,8634$ & 0,6554 \\
\hline & cuerda luego suelta & 3,7263 & $1,7558-7,9084$ & 1,4307 \\
\hline & caseta luego suelta & 1,4616 & $0,4119-5,1862$ & 0,9444 \\
\hline \multirow[t]{4}{*}{ método suministro } & balde & - & - & - \\
\hline & tetero & 2,8383 & $1,0086-7,9871$ & 1,4983 \\
\hline & milkbar & 2,8137 & $1,0855-7,2933$ & 1,3673 \\
\hline & tetero luego milkbar & 1,8596 & $0,8856-3,9048$ & 0,7039 \\
\hline \multirow[t]{2}{*}{ concentrado terneros } & no & - & - & - \\
\hline & si & 0,0553 & $0,0370-0,0825$ & 0,0113 \\
\hline
\end{tabular}

Por otro lado se encontró que el suministro de leche de descarte era un factor de riesgo para presentación de DNB (datos propios) y como la leche de descarte se compone de la proveniente de vacas con diferentes grados de mastitis, de calostro y de leche con residuos de medicamentos, puede relacionarse con el resultado obtenido en el presente estudio. Sin embargo, otros autores reportaron que el consumo de leche de descarte era un factor asociado a menor riesgo ${ }^{1}$; tal discrepancia demandaría la realización de estudios prospectivos aclaratorios.

Los hijos de vacas de raza Holstein tuvieron un menor riesgo de enfermar de DNB comparado con cualquiera de las otras razas en el estudio. El total de las vacas Holstein fue de 508 correspondiendo al 81,9\% de la población.

Factores asociados al ternero y su manejo. La Tabla 3 indica que existió mayor riesgo de DNB en los animales de raza diferente a la Holstein. Este resultado es contrario a lo encontrado en la misma zona (datos propios); sin embargo las características de manejo de las fincas y las razas presentes fueron diferentes en los dos estudios. En el presente trabajo un alto porcentaje de terneros $(79,2 \%)$ fue de raza Holstein, mientras que el número de animales pertenecientes a otras razas fue significativamente menor.
En Suiza se reportó que los animales de carne presentaron mayor riesgo de morir en la primer semana de vida comparado con los de leche y se atribuyó este hallazgo al mejor control que hay en las lecherías sobre el consumo de calostro de los terneros ${ }^{2}$.

En el presente estudio, los machos presentaron mayor probabilidad de enfermar $(\mathrm{OR}=29,1)$ que las hembras, a pesar de que el total de machos fue tan solo de $51(8,2 \%)$ y el de hembras $569(91,8 \%)$. En otros ensayos se determinó que los machos tuvieron mayor riesgo de morir que las hembras $(\mathrm{OR}=1,3)$, sugiriendo que el mayor peso al nacimiento de los machos podría predisponer a una mayor posibilidad de distocias ${ }^{2}$. En el estudio actual hubo asociación con el peso pero no con la presencia de distocias.

El modelo indicó que los terneros de peso medio (entre 39 y $45 \mathrm{~kg}$ ) tuvieron un menor riesgo de enfermar con DNB comparado con terneros más livianos o más pesados. Sin embargo se encontró una mayor probabilidad de enfermar cuando el peso fue inferior $(\mathrm{OR}=2,16)$ que cuando el peso fue superior $(\mathrm{OR}=1,7)$ al rango medio.

Este hecho podría deberse a que terneras de mayor peso podrían sufrir distocia, pero los partos distócicos no se hallaron como un factor de riesgo en el estudio, mientras que animales de muy bajo peso, pueden indicar partos prematuros o hijos de madres primerizas, que podrían predisponer a estos animales a DNB. Sin 
embargo estas características no fueron estadísticamente significativas como factores de riesgo. Los partos distócicos se han asociado con mayor riesgo de enfermar y morir debido al estrés del ternero, que reduce la respuesta a los patógenos, al menor vigor, al reducido consumo de calostro y la mayor permanencia del animal en decúbito, que favorece mayor contacto con material fecal $2,12,20$.

El sistema de manejo del ternero después de la separación de la madre presentó un impacto estadísticamente significativo en la presentación de DNB: hubo menor probabilidad cuando los terneros se manejaron en sistema de estaca y cuerda que en los otros sistemas de manejo. Para el manejo en terneril, cuerda-luego suelta y caseta-luego suelta, las probabilidades de enfermar fueron mayores $(\mathrm{OR}=2,1, \mathrm{OR}=3,7$ y $\mathrm{OR}=1,5$ respectivamente).

El manejo en sistema de cuerda y estaca se recomendaría por ser el método en el cual se observó menor riesgo de desarrollar DNB. Este resultado se convierte en un aporte importante que podría implementarse en las fincas. Esta característica protectora podría deberse a que los animales tienen menor posibilidad de tener contacto directo con áreas contaminadas con heces de animales afectados, generalmente son ubicados en potreros exclusivos para terneros, son removidos frecuentemente para que tengan disponibilidad de forraje fresco, limpio y con el menor tiempo de permanencia en cada área disminuye el reto microbiológico.

Teniendo en cuenta las diferencias en los sistemas de manejo utilizados en otros países, no se encontraron reportes previos con resultados similares. En estudios previos se ha investigado la relación existente con la enfermedad pero evaluando densidades y tamaños de los alojamientos en sistemas de estabulación ${ }^{22}$. Así, se encontró que los animales alojados sobre pisos de concreto tenían 8,9 veces más riesgo de enfermar que en otras superficies ${ }^{6}$. También se halló que los terneros alojados en terneriles abiertos expuestos a inclemencias climáticas, tuvieron mayor riesgo que los alojados en terneriles cerrados y que el uso de paja en la cama disminuyó el riesgo de heces fluidas en los terneros ${ }^{1}$, resultados que no son comparables por las diferencias en el manejo de las ganaderías en la zona bajo estudio.

Las prácticas de desinfección de ombligo estuvieron asociadas con la presentación de DNB y se evidenció mayor riesgo en fincas que no lo hacían $(\mathrm{OR}=6)$ o que lo hacían con eterol $(\mathrm{OR}=9,6)$. La mejor práctica resultó ser la utilización de productos yodados. En experiencias propias anteriores, la práctica de desinfección de ombligo resultó ser un factor de protección contra la mortalidad, pero estadísticamente no lo fue contra la morbilidad general.

Los terneros que consumieron calostro directamente de la madre presentaron menor riesgo de sufrir DNB que cuando se utilizaron como fuente, banco de calostro o calostro de una vaca diferente de la madre; sin embargo, estos resultados no son concluyentes dado que el número de animales que recibieron calostro de una fuente diferente a la madre fue bajo, tan solo 5 animales. En otros trabajos el grado de asociación fue 23\% menor para terneros que consumieron calostro exclusivamente de la propia madre $(\mathrm{OR}=0,77)$ comparado con otras fuentes ${ }^{10}$.

En cuanto al método de suministro de leche se observó menor riesgo para los terneros alimentados con balde cuando se comparó con la administración con tetero $(\mathrm{OR}=2,8)$, con milkbar $(\mathrm{OR}=2,8)$ y tetero luego milkbar $(\mathrm{OR}=1,86)$. El menor riesgo del balde coincide con el sistema de manejo, ya que en el sistema de estaca y cuerda generalmente se suministra la leche en balde. Resultados opuestos se reportaron en la misma zona (datos propios), donde el uso de balde representó mayor riesgo de morbilidad por DNB (OR=20,5 $\mathrm{p}=0,0006)$.

Los terneros alimentados con concentrado mostraron menor riesgo de presentar diarrea $(\mathrm{OR}=0,05)$, en coincidencia a lo reportado en anteriores investigaciones ${ }^{12}$. Otro estudio demostró que el suministro de concentrado a los terneros de mayor edad frente al suministro del mismo a temprana edad, representa un riesgo para mortalidad en terneros de 0 a 90 días y sugiere que el consumo de concentrado estimula la diferenciación del epitelio de absorción del rumen a las papilas características y al suministrarlo a una edad temprana pueden mejorar las condiciones de crecimiento e incrementar la resistencia hacia patógenos ${ }^{23}$.

En el análisis estadístico no se encontró que la falla en la transferencia pasiva (FTP) de inmunoglobulinas fuera un factor asociado a la enfermedad, observación similar a la reportada por otros autores ${ }^{24}$, aunque sí hubo menor porcentaje de absorción completa en los animales enfermos $(75,8 \%)$ que en los sanos $(78,2 \%)$. Los resultados de estudios previos muestran que la FTP de inmunoglobulinas, medida como proteínas plasmáticas totales, es un factor de riesgo de enfermedad (datos propios) y específicamente para diarrea ${ }^{11}$.

\section{CONCLUSIONES}

Los factores de riesgo asociados a la presentación de DNB encontrados en el estudio son: los terneros de fincas medianas y pequeñas, el uso de fertilizantes diferentes a los químicos, prácticas de suplementación, vacunación contra complejo neonatal bovino, raza diferente a la Holstein en madres y terneros, ser machos, tener peso menor de $39 \mathrm{~kg}$ o mayor de $45 \mathrm{~kg}$, desinfección de ombligos con productos diferentes a los yodados, permanencia post-separación de la madre en sistema diferente a cuerda y estaca y método de suministro diferente al uso de balde.

En contraposición, los factores de protección fueron: el suministro de concentrado a los terneros, aplicación de vacunas contra leptospirosis en la finca, complejo respiratorio bovino y vacuna triple bovina, prácticas de desparasitación con albendazoles a terneros y animales adultos, aplicación de terapia de secado y asistencia técnica de veterinarios y zootecnistas. 
Agradecimientos. A Intervet Schering-Plough Animal Health por el apoyo financiero para el desarrollo del proyecto. A los laboratorios clínico, parasitológico, microbiológico y virológico de la Facultad de Medicina Veterinaria y Zootecnia de la Universidad Nacional de Colombia, por la colaboración prestada.

\section{REFERENCIAS}

1. Al Mawly J, Grinberg A, Prattley D, Moffat J, Marshall J, French N. 2015. Risk factors for neonatal calf diarrhoea and enteropathogen shedding in New Zealand dairy farms. The Vet $J$ 203: 155-160.

2. Bleul U. 2011. Risk factors and rates of perinatal and postnatal mortality in cattle in Switzerland. Livestock Sci 135 : 257-264.

3. Burdick N, Banta J, Neuendorff D, White J, Vann R, Laurenz J, Welsh T, Randel R. 2009. Interrelationships among growth, endocrine, immune, and temperament variables in neonatal Brahman calves. J Anim Sci 87: 32023210 .

4. Frank N, Kaneene J. 1993. Management risk factors associated with calf diarrhea in Michigan dairy herds. $J$ Dairy Sci 76: 1313-1323.

5. González D, Mozgovoj V, Bellido D, Fernandez F, Wigdorovitz A, Parreño V, Dus Santos M. 2010. Evaluation of a bovine rotavirus VP6 vaccine efficacy in the calf model of infection and disease. Vet Immunol \& Immunopathol 137: 155-160.

6. Gulliksen S, Jor E, Lie K, Hamnes I, Løken T, Åkerstedt J, Østerås O. 2009. Enteropathogens and risk factors for diarrhea in Norwegian dairy calves. J Dairy Sci 92: 5057-5066.

7. Hosmer D, Lemeshow S. 2000. Applied logistic regression (Wiley Series in Probability and Statistics), Ed. John Wiley \& Sons Inc., Canada.

8. Klein-Jöbstl D, Iwersen M, Drillich M. 2014. Farm characteristics and calf management practices on dairy farms with and without diarrhea: A case-control study to investigate risk factors for calf diarrhea. J Dairy Sci 97: 51105119.

9. Lee S, Jaekal J, Baev C, Chung B, Yun S, Gwak M, Noh G, Lee D. 2008. Enzyme-linked immunosorbent assay, single radial immunodiffusion, and indirect methods for the detection of failure of transfer of passive immunity in dairy calves. J Vet Int Med 22: 212-218.

10. Leslie K, Todd C. 2007. Health status of calves in North America and Scandinavia. Proceedings from the Conference Calf Management (Norway), p. 37-44.
11. Lorenz I. 2006. Diarrhoea of the young calf: an update. World Buiatrics Congress, Nice, France.

12. Lorino R, Daudin J, Robin S, Sanaa M. 2005. Factors associated with time to neonatal diarrhoea in french beef calves. Prev Vet Med 68: 91-102.

13. Lundborg G, Oltenacu P, Maison D, Svensson E, Liberg P. 2003. Dam-related effects on heart girth at birth, morbidity and growth rate from birth to 90 days of age in Swedish dairy calves. Prev Vet Med 60: 175-190.

14. Mansfield M, Kuldau G. 2007. Microbiological and molecular determination of mycobiota in fresh and ensiled maize silage. Mycologia 99: 269-278.

15. Navarre C. 2000. Differentiation of gastrointestinal diseases of calves. Food Anim Pract 6: 37-57.

16. Noordhuizen J. 1997. Application of quantitative methods in veterinary epidemiology, 1st ed., Wageningen Pers, Wageningen, The Netherlands.

17. Pare J, Thurmond M, Gardner I, Picanso J. 1993. Effect of birthweight, total protein, serum igg and packed cell volume on risk of neonatal diarrhea in calves on two California dairies. Cannadian J Vet Res 57: 241-246.

18. Parreño V, Bejara C, Vagnozzia A, Barrandeguya M, Costantinia V, Craig M, Yuanb L, Hodginse D, Saifb L, Fernández F. 2004. Modulation by colostrum-acquired maternal antibodies of systemic and mucosal antibody responses to rotavirus in calves experimentally challenged with bovine rotavirus. Vet Immunol \& Immunopathol 100 : 7-24.

19. Peña N. 1988. La diarrea neonatal aguda indiferenciada en ganaderías del Piedemonte Llanero. Disciplinas Pecuarias 4: 11-12.

20. Sivula N, Ames T, Marsh W. 1996. Management practices and risk factors for morbidity and mortality in Minnesota dairy heifer calves. Prev Vet Med 27: 173-182.

21. Svensson C, Hultgren J, Oltenacu P. 2006. Morbidity in 3-7-month old dairy calves in south-western Sweden, and risk factors for diarrhoea and respiratory diseases. Prev Vet Med 74: 162-179.

22. Svensson C, Liberg P. 2006. The effect of group size on health and growth rate of Swedish dairy calves housed in pens with automatic milk-feeders. Prev Vet Med 73: 43-53.

23. Torsein M, Lindberg A, Sandgren CH, Waller KP, Törnquist M, Svensson C. 2011. Risk factors for calf mortality in large Swedish dairy herds. Prev Vet Med 99: 136-147.

24. Trotz L, Martin S, Leslie K, Duffield T, Nydam D, Peregrine A. 2007. Calf level risk factors for neonatal diarrhea and shedding of Cryptosporidium parvum in Ontario dairy calves. Prev Vet Med 82: 12-28. 